\title{
Correction: PD-L1 in small bowel adenocarcinoma is associated with etiology and tumor-infiltrating lymphocytes, in addition to microsatellite instability
}

Paolo Giuffrida - Giovanni Arpa · Federica Grillo - Catherine Klersy (1D - Gianluca Sampietro • Sandro Ardizzone • Paolo Fociani - Roberto Fiocca - Giovanni Latella - Fausto Sessa - Antonietta D'Errico - Deborah Malvi • Claudia Mescoli · Massimo Rugge · Gabriella Nesi · Stefano Ferrero • Daniela Furlan (1) - Gilberto Poggioli • Fernando Rizzello • Maria C. Macciomei - Donatella Santini - Umberto Volta • Roberto De Giorgio - Giacomo Caio • Antonio Calabrò - Carolina Ciacci (ib) Maria D'Armiento - Aroldo Rizzo - Gaspare Solina • Michele Martino • Francesco Tonelli • Vincenzo Villanacci - Renato Cannizzaro • Vincenzo Canzonieri · Ada M. Florena • Livia Biancone • Giovanni Monteleone - Roberto Caronna - Antonio Ciardi · Luca Elli · Flavio Caprioli • Maurizio Vecchi • Renata D'Incà · Fabiana Zingone · Anna D'Odorico • Marco Vincenzo Lenti · Barbara Oreggia •

Luca Reggiani Bonetti - Marco Astegiano - Elena Biletta · Laura Cantoro · Antonino G. Giannone · Augusto Orlandi • Claudio Papi - Vittorio Perfetti - Erica Quaquarini - Giancarlo Sandri - Marco Silano - Paolo Usai - Valeria Barresi • Rachele Ciccocioppo - Ombretta Luinetti - Paolo Pedrazzoli • Andrea Pietrabissa - Alessandra Viglio - Marco Paulli • Gino R. Corazza Enrico Solcia · Alessandro Vanoli • Antonio Di Sabatino

Published online: 4 March 2020

(c) The Author(s), under exclusive licence to United States \& Canadian Academy of Pathology 2020

\section{Correction to: Modern Pathology} https://doi.org/10.1038/s41379-020-0497-0

Author 'Marco Vincenzo Lenti' was listed in the original article as given name: 'Marco', family name: 'Vincenzo
Lenti'. However, this should be listed as given name: 'Marco Vincenzo', family name: 'Lenti'. This has been addressed by means of this correction article as well as an update to the original article. 